\title{
ASYMPTOTIC BEHAVIOR OF SOLUTIONS OF NONLINEAR DIFFERENTIAL EQUATIONS
}

\author{
BY \\ RICHARD K. MILLER( ${ }^{1}$ )
}

I. Introduction. In this paper we shall consider the system of $n$ first order ordinary differential equations of the form

$$
x^{\prime}=P(t, x)+R(t, x)+G(t, x) \quad\left({ }^{\prime}=\frac{d}{d t}\right),
$$

where we assume throughout that the following hypotheses are satisfied:

$\left(\mathrm{H}_{1}\right) R(t, x)$ and $G(t, x)$ are defined and continuous on $I \times D(I=$ $\{t ; 0 \leqq t<\infty\}$ and $D$ an open subset of $n$-dimensional Euclidean space $R^{n}$ ). in $t$.

$\left(\mathrm{H}_{2}\right) P(t, x)$ is defined and continuous on $R^{1} \times D$ and is almost periodic

$\left(\mathrm{H}_{3}\right) G(t, x)$ is integrable in the following sense: If $y(t)$ is any continuous bounded function from $I$ into $D$, then $|G(t, y(t))|$ is of class $L_{1}[0, \infty)$.

Let $Q$ be a fixed subset of $D$ such that $Q$ is closed in the topology of $D$. We shall assume that $R(t, x)$ satisfies the following two properties with respect to the set $Q$.

$\left(\mathrm{H}_{4}\right)$ For each $x$ in the set $Q, R(t, x) \rightarrow 0$ as $t \rightarrow \infty$ uniformly on compact subsets of $Q$.

$\left(\mathrm{H}_{5}\right)$ For each $\epsilon>0$ and each $x$ in $Q$ there exist numbers $\delta>0$ and $T$ $\geqq 0$, depending only on $\epsilon$ and $x$, such that for all $y \in D$ with $|x-y|<\delta$ and all $t \geqq T$ one has $|R(t, x)-R(t, y)|<\epsilon$.

Under the assumptions $\left(\mathrm{H}_{1}\right)-\left(\mathrm{H}_{5}\right)$ the system (PE) may be considered as a perturbation of the almost periodic system

$$
x^{\prime}=P(t, x) .
$$

In §II we shall be concerned with the asymptotic behavior of solutions $x(t)$ of (PE) which approach $Q$ as $t \rightarrow \infty$. We consider two cases: $x(t)$ bounded (Theorem 1) and $x(t)$ unbounded (Theorem 2). Since applications of these theorems are of interest, we present these in \$§II and III and postpone the somewhat lengthy proofs of Theorems 1 and 2 as well as the proofs of two preliminary lemmas to $\S \mathrm{V}$ and VI. In $\S I V$ we discuss

Presented to the Society, April 25, 1964 under the title The asymptotic behavior of asymptotically almost periodic differential equations; received by the editors May 18, 1964.

(1) The preparation of this manuscript was supported by the National Science Foundation under contract number NSF-G-4335. The author would like to express his appreciation to Dr. J. A. Nohel and to Dr. T. Yoshizawa for their many valuable suggestions. This paper is a portion of the author's doctoral dissertation written under the supervision of Dr. J. A. Nohel. 
some examples which illustrate the use of Theorem 1.

Barbašin and Krasovskii [2] obtain results on the asymptotic behavior of autonomous systems by combining the method of Liapunov with the notion of an invariant set. LaSalle [8] generalizes their results to periodic systems. Krasovskii [6] considers instability of periodic systems using similar methods. In \$III below we obtain generalizations of these results usirg Theorem 1 of this paper and some results from Liapunov theory.

The following conventions will be used in this paper: A function $y(t)$ defined on an interval $I_{0}$ with values in $D$ will be called bounded if there is a compact subset $D^{*} \subset D$ such that $y(t) \in D^{*}$ for all $t \in I_{0}$. If

$$
y=\left(y_{1}, y_{2}, y_{3}, \cdots, y_{n}\right)
$$

is a point of $R^{n}$, the norm of $y$ is $|y|=\sum_{i=1}^{n}\left|y_{i}\right|$. If $x$ is a point and $A$ a subset of $R^{n}$, then $d(x, A)=\inf \{|x-a| ; a \in A\}$; moreover, $N(A, \epsilon)$ $=\{x ; d(x, A)<\epsilon\}$ is the $\epsilon$-neighborhood of the set $A$. The symbol $x\left(t, t_{0}, x_{0}\right)$ will denote a function of $t$ such that $x\left(t_{0}, t_{0}, x_{0}\right)=x_{0}$.

If $y(t)$ is a continuous function on an interval $T \leqq t<\infty$ " with values in $R^{n}$, we recall that the positive limit set $\Gamma^{+}(y(t))$ is the set of all points $z \in R^{n}$ such that corresponding to each $z$ there is a sequence $t_{m} \rightarrow \infty$ as $m \rightarrow \infty$ with $y\left(t_{m}\right) \rightarrow z$. It is known (cf. [4, Chapter 16] or [7, p. 58]) that when $y(t)$ is bounded on an interval $T \leqq t<\infty$, the set $\Gamma^{+}(y(t))$ is nonempty, compact and $y(t) \rightarrow \Gamma^{+}(y(t))$ as $t \rightarrow \infty$, i.e., for each $\epsilon>0$ there is a $T(\epsilon)>0$ such that $y(t) \in N\left(\Gamma^{+}(y(t)), \epsilon\right)$ for all $t \geqq T(\epsilon)$. The reader may note that $\Gamma^{+}(y(t))$ is usually defined only if $y(t)$ is a solution of some differential equation. However, the definition of $\Gamma^{+}(y(t))$ and the proofs of the properties stated above depend only on the continuity and boundedness of $y(t)$.

The following remarks concern the hypothesis $\left(\mathrm{H}_{2}\right)$ :

Definition. If $P(t, x) \in C\left(R^{1} \times D\right)$, we shall call $P(t, x)$ almost periodic in $t$ if and only if the following two conditions are satisfied:

(i) For each fixed $x \in D, P(t, x)$ is almost periodic in the sense of $H$. Bohr (cf. [3] or [5, p. 281]) as a function of $t$.

(ii) For each compact subset $D^{*} \subset D, P(t, x)$ is uniformly continuous on the set $R^{1} \times D^{*}$.

It is known (cf. [3] or [5]) that if $P(t, x)$ is almost periodic in $t$ and $D^{*}$ is any compact subset of $D$, then $|P(t, x)|$ is bounded on $R^{1} \times D^{*}$. Further, if $\left\{h_{m}\right\}$ is any real sequence, there is a subsequence $\left\{h_{m k}\right\}$ and a function $P^{*}(t, x)$ which is almost periodic in $t$ such that

$$
P\left(t+h_{m k}, x\right) \rightarrow P^{*}(t, x) \quad \text { as } k \rightarrow \infty,
$$

uniformly for $t \in R^{1}$ and $x$ on compact subsets of $D$.

If $P(t, x)$ is almost periodic in $t$, let $\operatorname{Tr}(P)$ be the set of all translate 
functions, i.e., $\operatorname{Tr}(P)=\{P(t+h, x) ;-\infty<h<\infty\}$. Let $\operatorname{Cl}(P)$ be the closure (in the sense of (1.1)) of $\operatorname{Tr}(P)$. Using (1.1) we see that $\operatorname{Cl}(P)$ is a compact metric space of functions $P^{*}(t, x)$ which are almost periodic in t. It is easy to prove that for any fixed $P^{*} \in \mathrm{Cl}(P)$, the set $\operatorname{Tr}\left(P^{*}\right)$ $=\left\{P^{*}(t+h, x) ;-\infty<h<\infty\right\}$ is dense in $\mathrm{Cl}(P)$. In the sequel we shall generalize the notion of an invariant set to almost periodic systems by using the space $\mathrm{Cl}(P)$.

II. Perturbed almost periodic systems.

A. Bounded solutions of perturbed almost periodic systems. The main result of this paper may now be stated as follows:

Theorem 1. Let $\left(\mathrm{H}_{1}\right)-\left(\mathrm{H}_{5}\right)$ be satisfied for a fixed set $Q$. Let $x(t)$ be a solution of (PE) which is defined and bounded on an interval $t_{0} \leqq t<\infty$. Let $x(t) \rightarrow Q$ as $t \rightarrow \infty$. Then for each point $z \in \Gamma^{+}(x(t))$ there exists a function $P^{*} \in \mathrm{Cl}(P)$, a sequence $t_{m} \rightarrow \infty$ as $m \rightarrow \infty$ and a function $y(t)$ such that

(a) $y(t)=z+\int_{0}^{t} P^{*}(s, y(s)) d s \quad(-\infty<t<\infty)$,

(b) $x\left(t+t_{m}\right) \rightarrow y(t)$ as $m \rightarrow \infty$ uniformly on compact subsets of $-\infty<t$ $<\infty$, and

(c) $P\left(t+t_{m}, x\right) \rightarrow P^{*}(t, x)$ as $m \rightarrow \infty$ uniformly for $-\infty<t<\infty$ and $x$ on compact subsets of $D$.

Further, if $\left\{t_{m}\right\}$ is any sequence such that $t_{m} \rightarrow \infty$ and $x\left(t_{m}\right) \rightarrow z$ as $m \rightarrow \infty$, then a subsequence of $\left\{t_{m}\right\}$ may be used in satisfying (a), (b), and (c).

B. Quasi-invariant sets. The conclusion of Theorem 1 suggests an invariance property of the set $\Gamma^{+}(x(t))$ with respect to the system $(E)$. This property is defined as follows:

Definition. If $A$ is a subset of $D$, then $A$ is called quasi-invariant with respect to the almost periodic system

$$
x^{\prime}=P(t, x)
$$

if and only if for each $z \in A$ there exists a function $P^{*} \in \mathrm{Cl}(P)$ and a solution $y(t)$ of the almost periodic system

$$
x^{\prime}=P^{*}(t, x)
$$

with $y(0)=z$, such that $y(t)$ exists and remains in a compact subset of $A$ for $-\infty<t<\infty$.

The content of Theorem 1 is that $\Gamma^{+}(x(t))$ is quasi-invariant with respect to system (E). Since a bounded solution $x(t)$ of system (PE) must approach its positive limit set as $t \rightarrow \infty$, we have immediately:

COROLLARY 1. Let the hypothesis of Theorem 1 hold. Let $Q_{0}$ be the largest quasi-invariant subset of $Q$ with respect to system (E). Then $x(t) \rightarrow Q_{0}$ as $t \rightarrow \infty$. 
Since the definition of quasi-invariance includes the notion of boundedness for $-\infty<t<\infty$, we also conclude the following:

Corollary 2. Let $\left(\mathrm{H}_{1}\right)-\left(\mathrm{H}_{5}\right)$ hold. If there exists a $P^{*} \in \mathrm{Cl}(P)$ such that all solutions of $y^{\prime}=P^{*}(t, y)$ which intersect $Q$ are unbounded then no bounded solution of (PE) approaches $Q$ as $t \rightarrow \infty$.

Proof. Let $\left\{h_{m}\right\}$ be a sequence such that $P\left(t+h_{m}, x\right) \rightarrow P^{*}(t, x)$ in the topology of $\mathrm{Cl}(P)$ as $m \rightarrow \infty$. Let $\left\{D_{m}\right\}$ be a sequence of compact subsets of $D$ such that $D_{1} \subset D_{2} \subset D_{3} \subset \cdots$ and the union of all $D_{m}$ is $D$. Pick a sequence $\left\{t_{m}\right\}$ such that $\left|P\left(t+t_{m}, x\right)-P(t, x)\right|<1 / m$ on $R^{1} \times D_{m}$. We may pick $t_{m}$ so large that $t_{m} \geqq m+\left|h_{m}\right|$. Thus $t_{m}+h_{m} \rightarrow \infty$ as $m \rightarrow \infty$ and

$$
P\left(t+t_{m}+h_{m}, x\right) \rightarrow P^{*}(t, x) \quad \text { in } \mathrm{Cl}(P) \text { as } m \rightarrow \infty \text {. }
$$

If there were a solution $x(t)$ of (PE) bounded for large $t$ and approaching $Q$ as $t \rightarrow \infty$, then $\left\{x\left(t_{m}+h_{m}\right)\right\}$ would have a limit point $z \in Q$. Applying Theorem 1 we see that there would be a solution $y(t)$ of $y^{\prime}=P^{*}(t, y)$ such that $y(0)=z$ and $y(t)$ is bounded on $-\infty<t<\infty$. Since no such $y(t)$ may exist, $x(t)$ cannot approach $Q$ and Corollary 2 is proved.

Yoshizawa [10] considers the case where $P(t, x)=H(x)$ is independent of $t$. Under the hypotheses of Theorem 1 above, he proves that for each point $z \in \Gamma^{+}(x(t))$ there is a solution $y(t)$ of the system $y^{\prime}=H(y)$ such that

(a) $y(t)=z+\int_{0}^{t} H(y(s)) d s(0 \leqq t<\infty)$ and

(b) $y(t)$ stays in a bounded subset of $\Gamma^{+}(x(t))$ for $0 \leqq t<\infty$.

From Yoshizawa's proof it follows that there is a sequence $t_{m} \rightarrow \infty$ as $m \rightarrow \infty$ such that $x\left(t+t_{m}\right) \rightarrow y(t)$ as $m \rightarrow \infty$ uniformly on compact subsets of $0 \leqq t<\infty$. Now in this case $\operatorname{Tr}(P)=\mathrm{Cl}(P)$ is the single function $H(x)$. It follows that Theorem 1 above generalizes Yoshizawa's result.

C. Unbounded solutions of perturbed almost periodic systems. Yoshizawa [11] also considers the case where $x(t) \rightarrow Q$ as $t \rightarrow \infty$ with $x(t)$ not necessarily bounded on an interval $t_{0} \leqq t<\infty$. His result can be generalized to perturbed almost periodic systems in the following way.

THEOREM 2. Let $\left(\mathrm{H}_{1}\right)-\left(\mathrm{H}_{5}\right)$ hold for a fixed set $Q$. Let $x(t)$ be a solution of (PE) on an interval $t_{0} \leqq t<\infty$ with $x(t) \rightarrow Q$ as $t \rightarrow \infty$. If $\Gamma^{+}(x(t))$ is not empty, then for each $z \in \Gamma^{+}(x(t))$ there is an $L>0$, a function $P^{*} \in \mathrm{Cl}(P)$, a function $y(t)$, and a sequence $t_{m} \rightarrow \infty$ as $m \rightarrow \infty$ such that

(a) $y(t)=z+\int_{0}^{t} P^{*}(s, y(s)) d s$ for $-L \leqq t \leqq L$,

(b) $x\left(t+t_{m}\right) \rightarrow y(t)$ as $m \rightarrow \infty$ uniformly on the interval $-L \leqq t \leqq L$, and

(c) $P\left(t+t_{m}, x\right) \rightarrow P^{*}(t, x)$ as $m \rightarrow \infty$ uniformly for $-\infty<t<\infty$ and $x$ on any compact subset of $D$.

D. The special case $P(t, x)$ periodic in $t$. In the special case where $P(t, x)$ is periodic in $t$, Theorem 1 has the following form. 
Theorem 3. Let the hypotheses of Theorem 1 hold. If $P(t, x)$ is periodic in $t$ of period $\theta$, then for each $z \in \Gamma^{+}(x(t))$ there is an $h(0 \leqq h<\theta)$, an increasing sequence $\left\{K_{m}\right\}$ of positive integers and a solution $y(t)$ of $(\mathrm{E})$ such that

(a) $y(t)=z+\int_{h}^{t} P(s, y(s)) d s(-\infty<t<\infty)$ and

(b) $x\left(t+K_{m} \theta\right) \rightarrow y(t)$ as $m \rightarrow \infty$ uniformly on compact subsets of $-\infty$ $<t<\infty$.

If $P(t, x)$ is periodic in $t$ of period $\theta$, then $\mathrm{Cl}(P)=\{P(t+h, x) ; 0 \leqq h<\theta\}$. Also if $t_{m} \rightarrow \infty$ as $m \rightarrow \infty$ is any sequence we may assume that $t_{m}$ has the form $t_{m}=h+K_{m} \theta+s_{m}$, where $s_{m} \rightarrow 0$ as $m \rightarrow \infty$. Using these two facts as well as Theorem 1 the reader will have no difficulty constructing a proof of Theorem 3.

E. Remarks on Theorems 1 and 2. In both Theorem 1 and its local extension, Theorem 2, no uniqueness assumptions are made.

Amerio [1, pp. 97-105] proves the following result.

Let $P(t, x) \in C(I \times D)$ be almost periodic in $t$. If there is a solution $x(t)$ of $x^{\prime}=P(t, x)$ which remains in a compact subset $D_{0} \subset D$ for $t$ on an interval $t_{0} \leqq t<\infty$, then for each $P^{*} \in \mathrm{Cl}(P)$ there is a solution $y(t)$ of $y^{\prime}=P^{*}(t, y)$ with $y(t) \in D_{0}$ for all $t \in R^{1}$.

Amerio's result may be obtained from Corollary 2 above. We note that if $Q=D$ and $R(t, x) \equiv G(t, x) \equiv 0$ then system (PE) reduces to system (E) and satisfies hypotheses $\left(\mathrm{H}_{1}\right)-\left(\mathrm{H}_{5}\right)$. In this special case Corollary 2 reduces to the following statement which is equivalent to Amerio's result:

If there is a function $P^{*} \in \mathrm{Cl}(P)$ such that all solutions of $y^{\prime}=P^{*}(t, y)$ are unbounded on $-\infty<t<\infty$, then all solutions of $x^{\prime}=P(t, x)$ become unbounded in their positive interval of existence.

F. On possible generalizations of Theorem 1. The conclusions of Theorems 1 and 2 are rather complicated statements relating the behavior of solutions $x(t)$ along sequences $\left\{t_{m}\right\}$ with particular solutions of certain equations $x^{\prime}=P^{*}(t, x)$. We might conjecture that the asymptotic behavior of $x(t)$ should have a simpler relationship to the system (E). For example we might conjecture that Theorem 1 is true for all $P^{*} \in \mathrm{Cl}(P)$. Alternately, we might conjecture that $z \in \Gamma^{+}(x(t))$ implies that for all solutions $y\left(t, t_{0}, z\right)$ of (E) with $t$ and $t_{0}$ sufficiently large we have $y\left(t, t_{0}, z\right)$ $\in \Gamma^{+}(x(t))$. The author is indebted to C. C. Conley for the following example which shows that both of these conjectures are false.

Consider the periodic system

$$
x^{\prime}=-y, \quad y^{\prime}=x+E(t) W(x, y) .
$$

The functions $E(t)$ and $W(x, y)$ are defined as follows. Fix a small number $d>0$. Let

$$
W(x, y)= \begin{cases}0 & \text { if } x^{2}+(y-1)^{2} \geqq 2 d, \\ 1 & \text { if } x^{2}+(y-1)^{2} \leqq d .\end{cases}
$$


Extend $W$ as a $C^{1}$ function to the entire plane.

The number $d$ may be taken so small that there is a number $e(0<e<\pi / 4)$ such that when $0 \leqq t \leqq \pi$ one has $\sin t \geqq 1-d$ if and only if $|t-\pi / 2|$ $\leqq e$. Then for $0 \leqq t \leqq \pi$ one has $\cos ^{2} t+(1-\sin t)^{2} \leqq 2 d$ if and only if $|t-\pi / 2| \leqq e$. Define

$$
E(t)= \begin{cases}0 & \text { if } \pi / 2-e \leqq t \leqq \pi / 2+e \\ 1 & \text { if } \pi / 2+2 e \leqq t \leqq(5 \pi) / 2-2 e\end{cases}
$$

Extend $E$ by requiring $E$ to be a continuous function, periodic of period $2 \pi$. System (2.1) is periodic in $t$, hence almost periodic in $t$. Let the sets $Q$ and $D$ both be the entire plane $R^{2}$. Since $R(t, x) \equiv G(t, x) \equiv 0$, system (2.1) satisfies hypotheses $\left(\mathrm{H}_{1}\right)-\left(\mathrm{H}_{5}\right)$. From the definition of $E(t)$ and $W(x, y)$ it is easy to see that $x(t)=\cos t, y(t)=\sin t$ is a bounded solution of $(2.1)$. The point $(x, y)=(0,1)$ is in the set $\Gamma^{+}(\cos t, \sin t)$.

For any $t_{0} \in R^{1}$, let $T_{0}=t_{0}(\bmod 2 \pi)$. Clearly, if $\left|T_{0}-\pi / 2\right| \geqq 2 e$, then the solution of $(2.1)$ passing through $(0,1)$ at time $t_{0}$ does not stay on $\Gamma^{+}(\cos t, \sin t)$. This shows that both of the above conjectures are false.

In Theorem 1 the hypothesis on $P(t, x)$ may be weakened. The properties of $P$ which are essential to the proof of Theorem 1 are:

(i) $|P(t, x)|$ is bounded on $R^{1} \times D^{*}$ for each compact subset $D^{*}$ of $D$, and

(ii) for each sequence $t_{m} \rightarrow \infty$ as $m \rightarrow \infty$ there is a subsequence $\left\{t_{l m}\right\}$ and a function $P^{*}(t, x) \in C\left(R^{1} \times D\right)$ such that for each $t \in R^{1}$,

$$
\int_{0}^{t}\left|P\left(s+t_{l m}, x\right)-P^{*}(s, x)\right| d s \rightarrow \infty
$$

uniformly on compact subsets of $x \in D$.

The second property is not a natural assumption and is generally rather difficult to verify. Therefore we shall always assume that $P(t, x)$ is periodic or almost periodic in $t$.

III. Almost periodic systems. In this section we shall prove two Liapunovtype results concerning system (E). These results generalize the results for periodic systems which were mentioned in $\S$ I. We shall first need to recall some notions and results of Liapunov theory.

Definition. Let $W(t, x) \in C(I \times D)$ and let $A$ be a subset of $D$. We shall call $W$ positive definite with respect to the set $A$ if and only if the following two conditions are satisfied:

(i) $W(t, x)=0$ if $x$ is in $A$.

(ii) For each $\epsilon>0$ and each compact subset $D^{*} \subset D$ there is a number $\delta=\delta\left(\epsilon, D^{*}\right)>0$ such that if $d(x, A) \geqq \epsilon$ and $x \in D^{*}$, then $W(t, x) \geqq \delta$ uniformly for all $t$ in $I$.

We shall call $W$ negative definite with respect to $A$ if and only if $-W$ is positive definite with respect to $A$. 
We shall need the following two preliminary lemmas. For reference, these lemmas are stated immediately below, though their proofs are postponed until §VI.

Lemma 1 concerns systems of the form

$$
x^{\prime}=F(t, x)+G(t, x), \quad \text { with } F \text { and } G \in C(I \times D) \text {. }
$$

Lemma 1. Suppose $V(t, x)$ is continuous on $I \times D$ and is locally Lipschitzian in $x$. Let both $F(t, x)$ and $V(t, x)$ be bounded on sets $I \times D^{*}$, for each compact subset $D^{*}$ of $D$. Let $G$ be integrable in the sense of hypothesis $\left(\mathrm{H}_{3}\right)$. Suppose the derivative $V$ with respect to system (3.1) in the sense of Yoshizawa [10] satisfies $V(t, x) \geqq W(x)$, where $W$ is positive definite with respect to $A \subset D$. Then all bounded solutions of (3.1) tend to $A$ as $t \rightarrow \infty$.

Lemma 2. If $V(t, x) \in C\left(R^{1} \times D\right)$ is almost periodic in $t$, then the func$W(x)=\inf \{V(t, x) ;-\infty<t<\infty\}$ is continuous in $x$.

The first main result of this section may be stated as follows:

Theorem 4. Let $P(t, x)$ be almost periodic in $t$. Suppose there is a nonnegative function $V(t, x) \in C\left(R^{1} \times D\right)$ such that

(i) $V(t, x)$ is locally Lipschitzian in $x$ and almost periodic in $t$, and

(ii) The derivative $\dot{V}$ with respect to system (E) is a nonpositive function which is almost periodic in $t$.

Let $A=\left\{x \in D\right.$; $\left.\inf _{-\infty<t<\infty}-\dot{V}(t, x)=0\right\}$ and let $A_{0}$ denote the largest quasi-invariant subset of $A$ with respect to system (E). Then all bounded solutions of (E) approach $A_{0}$ as $t \rightarrow \infty$.

Proof. Let $x(t)$ be a bounded solution of $(\mathrm{E})$. Let

$$
W(x)=\inf \{-\dot{V}(t, x) ;-\infty<t<\infty\} .
$$

By Lemma $2, W$ is continuous on $D$. Since $W(z) \geqq 0, W$ is positive definite with respect to the set $A$. By a result of Yoshizawa [10, p. 382] all bounded solutions of (E) approach $A$ as $t \rightarrow \infty$.

For $Q=D$ and $R(t, x) \equiv G(t, x) \equiv 0$, system (PE) reduces to system (E) and at the same time satisfies $\left(\mathrm{H}_{1}\right)-\left(\mathrm{H}_{5}\right)$. It follows from Corollary 1 above that the solution $x(t)$ approaches $A_{0}$ as $t \rightarrow \infty$. This proves Theorem 4.

Note that if $x$ is any point of the positive limit set of the bounded solution $x(t)$, then one may use Theorem 1 above to obtain corresponding functions $y(t)$ and $P^{*}(t, x)$ as well as a sequence $\left\{t_{m}\right\}$. Since $V(t, x)$ is almost periodic in $t$ it may be assumed that $V\left(t+t_{m}, x\right) \rightarrow V^{*}(t, x)$ as $m \rightarrow \infty$ for some $V^{*} \in \operatorname{Cl}(V)$. Since $V(t, x(t)) \geqq 0$ and $\dot{V}(t, x(t)) \leqq 0$, the limit $V(t, x(t)) \rightarrow V_{0}$ exists as $t \rightarrow \infty$. If one notes that

$$
V\left(t+t_{m}, x\left(t+t_{m}\right)\right) \rightarrow V^{*}(t, y(t))
$$


as $m \rightarrow \infty$, it is clear that $V^{*}(t, y(t))=V_{0}$ is constant for $t \in R^{1}$. Thus for all $t \in R^{1}$,

$$
\limsup _{h \rightarrow 0^{+}} \frac{1}{h}\left(V^{*}(t+h, y(t+h))-V^{*}(t, y(t))\right)=0 .
$$

Using (3.2) and Theorem 4 we may prove the following corollary.

Corollary 3. Let $P(t, x)$ be periodic in $t$ of period $\theta$. Suppose there is a function $V(t, x) \in C^{1}\left(R^{1} \times D\right)$ with $V(t+\theta, x)=V(t, x) \geqq 0$ and with the derivative $\dot{V}$ with respect to system (E) nonpositive. Let

$$
B=\left\{\left(t_{0}, x_{0}\right) ; \dot{V}\left(t_{0}, x_{0}\right)=0\right\}
$$

and let $B_{0}$ be the largest subset of $B$ such that for each $\left(t_{0}, x_{0}\right) \in B_{0}$ there is $a$ solution $y(t)$ of (E) with $y\left(t_{0}\right)=x_{0}, y(t)$ bounded on $-\infty<t<\infty$, and $(t, y(t)) \in B_{0}$ for $-\infty<t<\infty$. Let $B_{x}=\left\{x ;\left(t_{0}, x_{0}\right) \in B_{0}\right.$ for some $\left.t_{0}\right\}$. Then all bounded solutions of (E) approach $B_{x}$ as $t \rightarrow \infty$.

Proof. Since $P$ is periodic in $t$ the set $A$ defined in Theorem 4 is the same as $\left\{x_{0} ; \dot{V}\left(t_{0}, x_{0}\right)=0\right.$ for some $\left.t_{0} \in R^{1}\right\}$. Also the function $V^{*}$ of (3.2) has the form $V^{*}(t, x)=V(t+h, x)$ for some $h$ in the range $0 \leqq h<\theta$. Therefore the conclusion of Theorem 4 together with (3.2) above imply the conclusion of Corollary 3 .

Corollary 3 generalizes LaSalle's result in [8] in two ways. The first is that no uniqueness assumption is necessary. Secondly, in defining the set $B_{0}$ of Corollary 3 we require that $y(t)$ must be bounded on $-\infty<t<\infty$.

The second main result of this section may be formulated as follows:

Theorem 5. Let $P(t, x)$ be almost periodic in $t$. Suppose there is a function $V(t, x) \in C\left(R^{1} \times D\right)$ such that

(i) $V(t, x)$ is locally Lipschitzian in $x$ and almost periodic in $t$,

(ii) $V$ has an infinitely small upper bound, and

(iii) the derivative $\dot{V}$ with respect to system (E) is non-negative and almost periodic in $t$.

Let

$$
A=\left\{x ; \inf _{-\infty<t<\infty} \dot{V}(t, x)=0\right\} .
$$

Let $A_{0}$, the largest quasi-invariant subset of $A$ with respect to system (E), be empty or just consist of the origin. If $V\left(t_{0}, x_{0}\right)>0$ for some $\left(t_{0}, x_{0}\right)$, then for each compact subset $D^{*} \subset D$ and each solution $x(t)$ of $(\mathrm{E})$ with $x\left(t_{0}\right)=x_{0}$, there is a number $t_{1}=t_{1}\left(D^{*}, x(t)\right) \geqq t_{0}$ such that $x\left(t_{1}\right) \notin D^{*}$.

Proof. If the theorem is not true, there is a compact set $D^{*}$ and a solution $x(t)$ of (E) with $x\left(t_{0}\right)=x_{0}$ and $x(t) \in D^{*}$ for all $t \geqq t_{0}$.

Let $W(x)=\inf \{\dot{V}(t, x) ;-\infty<t<\infty\}$. From Lemma 2 and assump- 
tion (iii) it follows that $W$ is positive definite with respect to $A$. From Corollary 1 above it follows that $x(t) \rightarrow A_{0}$ as $t \rightarrow \infty$. Since $A_{0}$ cannot be empty (Theorem 1), $A_{0}$ is the origin. Thus $x(t) \rightarrow 0$ as $t \rightarrow \infty$.

From $V(t, x) \geqq 0$ it follows that $V(t, x(t)) \geqq V\left(t_{0}, x_{0}\right)$ for all $t \geqq t_{0}$. From assumption (ii) it follows that there is an $h>0$ such that if $|x| \leqq h$, then $|V(t, x)|<V\left(t_{0}, x_{0}\right)$ uniformly for $t$ in $R^{1}$. Therefore $|x(t)| \geqq h>0$ for all $t \geqq t_{0}$. This contradicts $x(t) \rightarrow 0$ as $t \rightarrow \infty$ and proves Theorem 5 .

We remark that $V(t, x)$ of Theorem 5 is not assumed to be definite or semidefinite of either sign. If there are points $x_{0}$ arbitrarily close to the origin and arbitrarily large numbers $t_{0}$ such that $V\left(t_{0}, x_{0}\right)>0$ then clearly the origin (if $x \equiv 0$ is indeed a solution of $(E)$ ) is unstable.

IV. Example. In the sequel we shall need a theorem on existence and boundedness of solutions of the general system

$$
x^{\prime}=F(t, x), \quad \text { with } F \in C(I \times D) .
$$

THEOREM 6. Suppose there is a non-negative scalar function $V(t, x)$ $\in C^{1}(I \times D)$ such that the derivative $\dot{V}(t, x)$ with respect to system (4.1) is nonpositive. Suppose for some $L>0$ the set $D(L)=\{(t, x) ; V(t, x)<L\}$ is bounded in the sense that there is a fixed compact set $D^{*} \subset D$ such that for all $T \geqq 0$ the intersection of $D(L)$ with the plane $t=T$ is contained in $D^{*}$. If $x(t)$ is any solution of (4.1) with $\left(t_{0}, x\left(t_{0}\right)\right) \in D(L)$ for some $t_{0} \geqq 0$, then $x(t)$ exists and remains bounded (in $D^{*}$ ) for all $t \geqq t_{0}$.

For the proof we note that if $\left(t_{0}, x\left(t_{0}\right)\right) \in D(L)$ then $V\left(t_{0}, x\left(t_{0}\right)\right)<L$. From $\dot{V}(t, x) \leqq 0$ it follows that $V(t, x(t))<L$ for all $t \geqq t_{0}$, i.e., $x(t) \in D^{*}$.

As an example of an application of Theorem 1 we consider a system similar to one considered by Levin and Nohel [9].

$$
\begin{aligned}
& x^{\prime}=-\sum_{i=0}^{n} a_{i}(t) z_{i}, \\
& z_{0}^{\prime}=b_{0}(t) f(x)+e_{0}(t), \\
& z_{i}^{\prime}=-h_{i}(t, x, z) z_{i}+b_{i}(t) f(x)+e_{i}(t) \quad(i=1,2,3, \cdots, n) .
\end{aligned}
$$

We assume the following concerning system (4.2):

(i) $a_{i}(t), b_{i}(t), e_{i}(t)$ and $f(x)$ are continuous on $R^{1}$ and $h_{i}(t, x, z)$ is continuous on $I \times R^{n+2}$.

(ii) $x f(x)>0$ if $x \neq 0$.

(iii) There is a constant $K>0$ such that $h_{i}(t, x, z) \geqq K$ for all $(t, x, z)$.

(iv) $h_{i}(t, x, z)$ is bounded in $t$ when $(x, z)$ is restricted to any compact subset of $R^{n+2}$.

(v) $e_{i}(t) \in L_{1}[0, \infty)$.

(vi) There are constants $c_{i}>0$ such that $a_{i}(t)=c_{i} b_{i}(t)$ for all $t \in R^{1}$.

(vii) Each $a_{i}(t)$ is almost periodic. 
(viii) There is a constant $M>0$ such that $\left|a_{0}(t)\right| \geqq M$ and $\left|a_{1}(t)\right| \geqq M$ for all $t \in R^{1}$.

We shall show that all bounded solutions of (4.2) tend to the origin as $t \rightarrow \infty$. To this end we define

$$
V(t, x, z)=\sum_{i=0}^{n}\left(c_{i} z_{i}^{2}\right) / 2+\int_{0}^{x} f(u) d u .
$$

Then as in [9] we find that

$$
\dot{V}(t, x, z) \leqq-W(z)+E_{1}^{\prime}(t) V(t, x, z)+E_{2}^{\prime}(t),
$$

where

$$
\begin{aligned}
& W(z)=K \sum_{i=1}^{n} c_{i} z_{i}^{2} \\
& E_{1}(t)=\int_{0}^{t} \sum_{i=0}^{n}\left|e_{i}(s)\right| d s,
\end{aligned}
$$

and

$$
E_{2}(t)=\int_{0}^{t} \sum_{i=0}^{n} c_{i}\left|e_{i}(s)\right| d s
$$

Define

$$
C=\lim _{t \rightarrow \infty} \exp \left(-E_{1}(t)-E_{2}(t)\right)
$$

and

$$
U(t, x, z)=\exp \left(-E_{1}(t)-E_{2}(t)\right)(V(t, x, z)+1) .
$$

Then

$$
\dot{U}(t, x, z) \leqq-C W(z) .
$$

Since $W(z)$ is positive definite on the set

$$
Q=\left\{(x, z) ; z_{1}=z_{2}=\cdots=z_{n}=0\right\},
$$

it follows from a result of Yoshizawa $[10$, p. 382] that all bounded solutions of (4.2) approach $Q$ as $t \rightarrow \infty$. From Corollary 1 above it follows that all bounded solutions of (4.2) approach the largest quasi-invariant subset of $Q$ with respect to the almost periodic system

$$
\begin{aligned}
& x^{\prime}=-a_{0}(t) z_{0}, \\
& z_{i}^{\prime}=b_{i}(t) f(x) \quad(i=0,1,2, \cdots, n) .
\end{aligned}
$$

We shall now show that the largest quasi-invariant subset of $Q$ is the 
origin. For suppose $\left(x^{0}, z^{0}, 0,0, \cdots, 0\right)$ is a point of this set. Then there is a bounded solution $(x(t), z(t))$ of a certain system

$$
\begin{aligned}
& x^{\prime}=-a_{0}^{*}(t) z_{0}, \\
& z_{i}^{\prime}=b_{i}^{*}(t) f(x) \quad(i=0,1,2, \cdots, n),
\end{aligned}
$$

where $a_{0}^{*} \in \mathrm{Cl}\left(a_{0}\right), b_{i}^{*} \in \mathrm{Cl}\left(b_{i}\right)$ and $a_{0}^{*}(t) \equiv c_{0} b_{0}^{*}(t)$.

Since the solution $(x(t), z(t))$ remains in $Q$, we must have $z_{i}(t) \equiv 0$ for $i=1,2,3, \cdots, n$. From assumption (viii) it follows that $c_{1}\left|b_{1}^{*}(t)\right| \geqq M$. Together with assumption (ii) and $z_{1}^{\prime}(t) \equiv b_{1}^{*}(t) f(x(t)) \equiv 0$ it follows that $x(t) \equiv 0$. Therefore

$$
z_{0}^{\prime}(t) \equiv c_{0} a_{0}^{*}(t) f(x(t)) \equiv c_{0} a_{0}^{*}(t) f(0) \equiv 0,
$$

and $z_{0}(t)=z^{0}$ is constant. Further, $x^{\prime}(t) \equiv-a_{0}^{*}(t) z_{0}$ and $\left|a_{0}^{*}(t)\right| \geqq M$ imply that $\dot{z}_{0}=0$. Thus $x^{0}=z^{0}=0$.

There is a neighborhood $D_{0}$ of the origin such that all solutions of (4.2) starting in $D_{0}$ at any time $t_{0} \geqq 0$ are bounded. To see this we use Theorem 6. Take $L>0$ such that

$$
\int_{0}^{x} f(u) d u+1<L / C
$$

implies that $|x|$ is bounded. That such an $L$ exists follows from assumption (ii). Then the set $D(L)=\{(t, x, z) ; U(t, x, z)<L\}$ is bounded in the sense of Theorem 6 above. Since $U$ is non-negative and $U$ is nonpositive, we may apply Theorem 6 .

Levin and Nohel assume that

$$
\lim _{|x| \rightarrow \infty} \int_{0}^{x} f(u) d s \rightarrow \infty .
$$

In this case the function $U(t, x, z) \rightarrow \infty$ as $|x|+|z| \rightarrow \infty$ uniformly for $t$ in $I$. It is known that in this case all solutions of (4.2) are bounded (cf. $[7$, p. 113]).

We now modify the previous example and consider any system of the form

$$
\begin{aligned}
& x^{\prime}=-\sum_{i=1}^{n} a_{i}(t) z_{i}-p(t) x, \\
& z_{i}^{\prime}=-h_{i}(t, x, z) z_{i}+b_{i}(t) f(x)+e_{i}(t) \quad(i=1,2,3, \cdots, n) .
\end{aligned}
$$

The functions $a_{i}, b_{i}, f, e_{i}$ and $h_{i}$ are assumed to satisfy the same assumptions as in the previous example. We assume $p(t) \geqq 0$ is almost periodic and is not identically zero. With $V$ defined by

$$
V(t, x, z)=\sum_{i=1}^{n}\left(c_{i} / 2\right) z_{i}^{2}+\int_{0}^{x} f(u) d u,
$$


we have

$$
\dot{V}(t, x, z) \leqq-W(z)+E_{1}^{\prime}(t) V(t, x, z)+E_{2}^{\prime}(t),
$$

where $W(z), E_{1}(t)$ and $E_{2}(t)$ are as in the previous example. Now let

$$
U(t, x, z)=\exp \left(-E_{1}(t)-E_{2}(t)\right)(V(t, x, z)+1) .
$$

Then

$$
\dot{U}(t, x, z) \leqq-C W(z) .
$$

From Yoshizawa's result $[10$, p. 382] it follows that all bounded solution of (4.5) approach the set $Q=\{(x, z) ; z=0\}$ as $t \rightarrow \infty$. By Corollary 1 , all bounded solutions approach $Q_{0}$, the largest quasi-invariant subset of $Q$, as $t \rightarrow \infty$. We shall now show that $Q_{0}$ is the origin. For let $\left(x_{0}, 0\right)$ be any point of $Q_{0}$. Then there is a system

$$
\begin{aligned}
& x^{\prime}=-p^{*}(t) x, \\
& z_{i}^{\prime}=b_{i}^{*}(t) f(x) \quad(i=1,2,3, \cdots, n)
\end{aligned}
$$

with $p^{*} \in \mathrm{Cl}(p)$ and $b_{i}^{*} \in \mathrm{Cl}\left(b_{i}\right)$ and there is a bounded solution $(x(t), z(t))$ of (4.6) such that $x(0)=x_{0}$ and $z(t) \equiv 0$. From (4.6) we see that

$$
x(t)=x_{0} \exp \left(-\int_{0}^{t} p^{*}(s) d s\right) .
$$

Since $p^{*}(t) \geqq 0$ is almost periodic, if $x_{0} \neq 0$ then $|x(t)| \rightarrow \infty$ as $t \rightarrow-\infty$. Therefore the set $Q_{0}$ is the origin. We also note that the considerations for the existence of bounded solutions of (4.2) carry over in a similar way to (4.5).

V. Proofs of Theorems 1 and 2. We shall need the following lemma which is easily established by a contradiction argument.

Lemma 3. If $Q^{*}$ is any compact subset of $Q$, then $\delta$ and $T$ of $\left(\mathrm{H}_{5}\right)$ may be chosen independently of $x$ in $Q^{*}$.

Proof of Theorem 1. Let $\left\{\epsilon_{m}\right\}$ be any positive null sequence. Let $D^{*}$ $\subset D$ be a compact set such that $x(t) \in D^{*}$ for all $t \geqq t_{0}$. Such a $D^{*}$ exists because $x(t)$ is bounded on the interval $t_{0} \leqq t<\infty$. Let $M$ be a bound for $|P(t, x)|$ on the set $t \geqq 0, x \in D^{*}$. Let $Q^{*}=Q \cap D^{*}$. Fix any $L>0$ and any point $z \in \Gamma^{+}(x(t))$.

By definition of $\Gamma^{+}(x(t))$ there exists a sequence $t_{m} \rightarrow \infty$ as $m \rightarrow \infty$ such that $x\left(t_{m}\right) \rightarrow z$. By possibly taking a subsequence we may assume that

$$
\left|x\left(t_{m}\right)-z\right|<\epsilon_{m} \text { for } m=1,2,3, \cdots .
$$

Using the boundedness of $x(t)$ as well as assumption $\left(\mathrm{H}_{3}\right)$ we may also assume that for all $t \geqq t_{m}-L$, 


$$
\int_{t}^{\infty}|G(t, x(t))| d t<\epsilon_{m}
$$

Using $\left(\mathrm{H}_{4}\right)$ we may assume that

$$
\sup \left\{|R(t, x)| ; x \in Q^{*} \text { and } t \geqq t_{m}-L\right\}<\epsilon_{m} .
$$

The compactness of $Q^{*}$ implies that for each $t \geqq t_{0}$ there is at least one point $x_{t} \in Q^{*}$ such that $d\left(x(t), Q^{*}\right)=\left|x(t)-x_{t}\right|$. By $\left(\mathrm{H}_{5}\right)$ and Lemma 3 there are numbers $\delta_{m}$ and $T_{m}$ such that if $x \in Q^{*}, t \geqq T_{m}$ and $|x-y|<\delta_{m}$, then $|R(t, x)-R(t, y)|<\epsilon_{m}$. Since $x(t) \rightarrow Q^{*}$ as $t \rightarrow \infty$ there exists $S_{m} \geqq T_{m}$ such that $d\left(x(t), Q^{*}\right)=\left|x(t)-x_{t}\right|<\delta_{m}$ for all $t \geqq S_{m}$. We may assume that $t_{m} \geqq S_{m}+L$. Thus

$$
|R(t, x(t))| \leqq\left|R(t, x(t))-R\left(t, x_{t}\right)\right|+\left|R\left(t, x_{t}\right)\right| \leqq 2 \epsilon_{m}
$$

for all $t \geqq t_{m}-L$.

We now define functions $x_{m}(t)$ on the interval $-L \leqq t \leqq L$ by $x_{m}(t)$ $=x\left(t+t_{m}\right)$. Since

$$
x(t)=x\left(t_{m}\right)+\int_{t_{m}}^{t}\{P(s, x(s))+R(s, x(s))+G(s, x(s))\} d s,
$$

we have

$$
\begin{aligned}
x_{m}(t)=x\left(t_{m}\right) & +\int_{0}^{t} P\left(s+t_{m}, x_{m}(s)\right) \\
& +\int_{t_{m}}^{t+t}\{R(s, x(s))+G(s, x(s))\} d s
\end{aligned}
$$

for $-L \leqq t \leqq L$.

Each of the functions $x_{m}(t)$ has its range in the compact set $D^{*}$. Thus the sequence $\left\{x_{m}(t)\right\}$ is uniformly bounded. The sequence is also equicontinuous. For take any $m$ and any $t_{1}$ and $t_{2}$ with $-L \leqq t_{1}<t_{2} \leqq L$; from (5.6) we see that

$$
\begin{aligned}
\left|x\left(t_{2}\right)-x\left(t_{1}\right)\right| \leqq & \int_{t_{1}}^{t_{2}}\left|P\left(s+t_{m}, x_{m}(s)\right)\right| d s+\int_{t_{m}+t_{1}}^{t_{m}+t_{2}}|R(s, x(s))| d s \\
& +\int_{t_{m}-L}^{\infty}|G(s, x(s))| d s .
\end{aligned}
$$

Using (5.2) and (5.4) we have

$$
\left|x\left(t_{2}\right)-x\left(t_{1}\right)\right| \leqq\left(M+2 \epsilon_{m}\right)\left(t_{2}-t_{1}\right)+\epsilon_{m} .
$$

Since $\epsilon_{m} \rightarrow 0$ as $m \rightarrow \infty$, the equicontinuity of $\left\{x_{m}(t)\right\}$ is proved.

There is a function $y(t)$ and a subsequence of $\left\{x_{m}(t)\right\}$ which we again index by $m$ such that 


$$
\left|x\left(t+t_{m}\right)-y(t)\right|=\left|x_{m}(t)-y(t)\right| \rightarrow 0 \quad \text { as } m \rightarrow \infty
$$

uniformly on $-L \leqq t \leqq L$. Using property (1.1) of $\S I$ above for the almost periodic function $P(t, x)$, there is a function $P^{*}(t, x) \in \mathrm{Cl}(P)$ and and another subsequence such that

$$
\left|P\left(t+t_{m}, x\right)-P^{*}(t, x)\right| \rightarrow 0 \quad \text { as } m \rightarrow \infty
$$

uniformly for $-\infty<t<\infty$ and $x$ on any compact subset of $D$.

From (5.1), (5.2), (5.4), (5.6) and the uniform convergence of the sequences $\left\{x_{m}(t)\right\}$ and $\left\{P\left(t+t_{m}, x\right)\right\}$ it follows that $y(t)$ has the form

$$
y(t)=z+\int_{0}^{t} P^{*}(s, y(s)) d s .
$$

Once (5.7) is established for arbitrary $L>0$ and arbitrary sequences $t_{m} \rightarrow \infty$ as $m \rightarrow \infty$ with $x\left(t_{m}\right) \rightarrow z$, the proof is completed as follows: For $L=1$ we may find a sequence $\left\{t_{1 m}\right\}$ which satisfies $(5.7)$ on $-1 \leqq t \leqq 1$. For $L=2$ we find a subsequence $\left\{t_{2 m}\right\}$ of $\left\{t_{1 m}\right\}$ which satisfies (5.7) on $-2 \leqq t \leqq 2$. We continue in this way for $L=3,4,5, \ldots$. Each of the sequences $\left\{t_{j m}\right\}$ defines the same function $P^{*}(t, x)$ and the same $y(t)$ (for as long as $y(t)$ is defined). Thus $y(t)$ may be defined for all of the interval $-\infty<t<\infty$. The subsequence satisfying conditions (a), (b) and (c) can be chosen as $t_{m}=t_{m m}$.

Proof of Theorem 2. Fix some point $z \in \Gamma^{+}(x(t))$. Pick $r_{0}>0$ such that $N_{0}=\left\{x ; d(x, z) \leqq r_{0}\right\} \subset D$. Define

$$
M_{0}=\sup \left\{|P(t, x)| ;-\infty<t<\infty, x \in N_{0}\right\}
$$

and

$$
M_{1}=1+\sup \{|R(t, z)| ; 0 \leqq t<\infty\} .
$$

Since $N_{0}$ is compact, $M_{0}$ is finite. In view of $\left(\mathrm{H}_{4}\right), M_{1}$ is also finite. By $\left(\mathrm{H}_{5}\right)$ there is a $T>0$ and a $\delta>0$ such that whenever $t \geqq T$ and $|x-z|$ $<\delta$, one has $|R(t, x)-R(t, z)|<1$. Let $r=\min \left(\delta, r_{0}\right)$. If $|x(t)-z|<r$ for any number $t \geqq T$, then $|R(t, x(t))|<M_{1}$.

Let $x^{*}(t)$ be a continuous bounded function defined on $0 \leqq t<\infty$ such that $x^{*}(t)=x(t)$ whenever $x(t) \in N_{0}$. We may pick a number $T_{1} \geqq T$ such that for all $t \geqq T_{1}$

$$
\int_{t}^{\infty}\left|G\left(s, x^{*}(s)\right)\right| d s<r / 3
$$

Let $L=r /\left(3 M_{0}+3 M_{1}\right)$. It is claimed that if there is a number $t_{0} \geqq T_{1}$ $+L$ with $\left|x\left(t_{0}\right)-z\right|<r / 3$, then $|x(t)-z|<r$ for $\left|t-t_{0}\right| \leqq L$. To see this suppose there is a $t_{1}$ with $t_{0}<t_{1}<t_{0}+L$ (or $t_{0}-L<t_{1}<t_{0}$ ) such that $|x(t)-z|<r$ for $t_{0}<t<t_{1}$ and $\left|x\left(t_{1}\right)-z\right|=r$. Since $x^{*}(t)=x(t)$ has the form (5.5) on the set $N_{0}$ we have 


$$
\begin{aligned}
r= & \left|x\left(t_{1}\right)-z\right| \leqq\left|x\left(t_{0}\right)-z\right|+\int_{t_{0}}^{t_{1}}\{|P(s, x(s))|+|R(s, x(s))|\} d s \\
& +\int_{t_{0}-L}^{\infty}\left|G\left(s, x^{*}(s)\right)\right| d s \\
& ⿱ r / 3+\left(M_{0}+M_{1}\right)\left|t_{1}-t_{0}\right|+r / 3<r .
\end{aligned}
$$

This contradiction proves the claim.

We now pick a sequence $t_{m} \rightarrow \infty$ as $m \rightarrow \infty$ such that

(i) $\left|x\left(t_{m}\right)-z\right|<r / 3 \quad(m=1,2,3, \cdots)$,

(ii) $x\left(t_{m}\right) \rightarrow z$ as $m \rightarrow \infty$, and

(iii) $T_{1}+L<t_{1}<t_{2}<t_{3}<\cdots$.

For such a sequence we know that $x(t) \in N(z, r) \subset N_{0}$ if $\left|t-t_{m}\right| \leqq L$. Define $x_{m}(t) \doteq x\left(t+t_{m}\right)$ for $-L \leqq t \leqq L$ and for $m=1,2,3, \ldots$. Let $Q^{*}=Q \cap N_{0}$. The proof may now be completed in the same way as the first part of the proof of Theorem 1 .

\section{Proof of Lemmas 1 and 2.}

Proof of Lemma 1. Let $x(t)$ be a solution of system (3.1) with range in a compact subset $D^{*}$ of $D$. Let $M$ be a bound for $|F(t, x)|$ and $|V(t, x)|$ on the set $t \geqq 0$ and $x \in D^{*}$.

If the lemma is not true, there is an $\epsilon>0$ and a sequence $t_{m} \rightarrow \infty$ as $m \rightarrow \infty$ such that $d\left(x\left(t_{m}\right), A\right) \geqq 2 \epsilon$. Let $T_{m}=t_{m}+\epsilon /(2 M)$. We may assume that $T_{m}<t_{m+1}$ for $m=1,2,3, \cdots$ and that

$$
\int_{t_{1}}^{\infty}|G(s, x(s))| d s<\epsilon / 2 .
$$

If $t_{m} \leqq t \leqq T_{m}$, then $\left|x(t)-x\left(t_{m}\right)\right|<\epsilon$ and $d(x(t), A) \geqq \epsilon$. By the positive definiteness of $W$, there is a $\delta>0$ such that if $x \in D^{*}$ and $d(x, A)$ $\geqq \epsilon$, then $\dot{V}(t, x) \geqq \delta$ uniformly for $t$ in $I$. Therefore

$$
\begin{aligned}
V\left(T_{m}, x\left(T_{m}\right)\right)-V\left(t_{1}, x\left(t_{1}\right)\right) & =\int_{t_{1}}^{T_{m}} \dot{V}(s, x(s)) d s \\
& \geqq \sum_{j=1}^{m} \int_{t_{j}}^{T_{j}} \dot{V}(s, x(s)) d s \\
& \geqq\left(\frac{\delta \epsilon}{2 M}\right) m .
\end{aligned}
$$

Thus

$$
M \geqq V\left(T_{m}, x\left(T_{m}\right)\right) \geqq V\left(t_{1}, x\left(t_{1}\right)\right)+\left(\frac{\delta \epsilon}{2 M}\right) m>M
$$

when $m$ is sufficiently large. This contradiction proves Lemma 1. 
Proof of Lemma 2. Pick any point $x_{0} \in D$ and any sequence $x_{m} \rightarrow x_{0}$ as $m \rightarrow \infty$. Let $w_{1}=\lim \sup W\left(x_{m}\right)(m \rightarrow \infty)$ and $w_{2}=\liminf W\left(x_{m}\right)(m \rightarrow \infty)$. Then $w_{1} \geqq w_{2}$. We now show that $w_{2} \geqq W\left(x_{0}\right) \geqq w_{1}$. From this it follows that $\lim W\left(x_{m}\right)=W\left(x_{0}\right) \quad(m \rightarrow \infty)$ exists.

Let $\left\{x_{1 m}\right\}$ be a subsequence of $\left\{x_{m}\right\}$ such that $W\left(x_{1 m}\right) \rightarrow w_{1}$ as $m \rightarrow \infty$. Pick $\epsilon>0$. Pick $\delta(\epsilon)$ using the uniform continuity of $V$ on the set $R^{1}$ $X\left\{x_{j} ; j=0,1,2, \ldots\right\}$ (note that $\left\{x_{j} ; j=0,1,2, \ldots\right\}$ is a compact set). There is an $M$ so large that $\left|x_{1 M}-x_{0}\right|<\delta(\epsilon)$. Therefore

$$
\left|V\left(t, x_{0}\right)-V\left(t, x_{1 M}\right)\right|<\epsilon \quad \text { uniformly for all } t \text { in } I .
$$

The number $M$ may be taken so that

$$
\left|w_{1}-W\left(x_{1 M}\right)\right|<\epsilon .
$$

Pick $s \in R^{1}$ such that

$$
\left|V\left(s, x_{0}\right)-W\left(x_{0}\right)\right|<\epsilon .
$$

From (6.1) and (6.3) it follows that

$$
\begin{aligned}
\left|W\left(x_{0}\right)-V\left(s, x_{1 M}\right)\right| & \leqq\left|W\left(x_{0}\right)-V\left(s, x_{0}\right)\right|+\left|V\left(s, x_{0}\right)-V\left(s, x_{1 M}\right)\right| \\
& <2 \epsilon .
\end{aligned}
$$

Thus

$$
W\left(x_{0}\right) \geqq V\left(s, x_{1 m}\right)-2 \epsilon \geqq W\left(x_{1 M}\right)-2 \epsilon .
$$

From (6.2) it follows that $W\left(x_{0}\right)>w_{1}-3 \epsilon$. Since $\epsilon>0$ is arbitrary it follows that $W\left(x_{0}\right) \geqq w_{1}$.

In order to show that $w_{2} \geqq W\left(x_{0}\right)$ pick an $\epsilon>0$ and an integer $M$ so large that

$$
\left|V\left(t, x_{M}\right)-V\left(t, x_{0}\right)\right|<\epsilon \text { uniformly for all } t \text { in } R^{1}
$$

and such that

$$
\left|W\left(x_{M}\right)-w_{2}\right|<\epsilon .
$$

Pick a number $s$ in $R^{1}$ such that

$$
\left|V\left(s, x_{M}\right)-W\left(x_{M}\right)\right|<\epsilon .
$$

Then

$$
\begin{aligned}
&\left|w_{2}-V\left(s, x_{0}\right)\right| \leqq\left|w_{2}-W\left(x_{M}\right)\right|+\left|W\left(x_{M}\right)-V\left(s, x_{M}\right)\right| \\
&+\left|V\left(s, x_{M}\right)-V\left(s, x_{0}\right)\right|<3 \epsilon, \\
& w_{2}>V\left(s, x_{0}\right)-3 \epsilon \geqq W\left(x_{0}\right)-3 \epsilon .
\end{aligned}
$$

Since $\epsilon>0$ is arbitrary, $w_{2} \geqq W\left(x_{0}\right) \geqq w_{1}$.

Since the point $x_{0}$ and the sequence $\left\{x_{m}\right\}$ are arbitrary, the assertion of the continuity of $W$ is proved. This completes the proof of Lemma 2 . 


\section{REFERENCES}

1. L. Amerio, Soluzioni quasi-periodiche, o limitate, di sistemi differenziali non lineari quasiperiodici, o limitati, Ann. Mat. Pura Appl. 39 (1955), 97-119.

2. E. A. Barbašin and N. N. Krasovskiĭ, On stability of motion in the large, Dokl. Akad. Nauk. SSSR 86 (1952), 453-456. (Russian)

3. A. S. Besicovitch, Almost periodic functions, Dover, New York, 1954.

4. E. A. Coddington and N. Levinson, Theory of ordinary differential equations, McGrawHill, New York, 1955.

5. N. Dunford and J. T. Schwartz, Linear operators, Part I, General theory, Pure and Applied Mathematics, Vol. 7, Interscience, New York, 1958.

6. N. N. Krasovskiî, Stability of motion, Stanford Univ. Press, Palo Alto, Calif., 1963.

7. J. P. LaSalle and S. Lefschetz, Stability by Liapunov's direct method with applications, Academic Press, New York, 1961.

8. J. P. LaSalle, Asymptotic stability criterion, Proc. Sympos. Appl. Math. Vol. 13, pp. 299307, Amer. Math. Soc., Providence, R. I., 1962.

9. J. J. Levin and J. A. Nohel, Global asymptotic stability for nonlinear systems of differential equations and applications to reactor dynamics, Arch. Rational Mech. Anal. 5 (1960), 194-211. 10. T. Yoshizawa, Asymptotic behavior of solutions of a system of differential equations, Contributions to Differential Equations 1 (1963), 371-387.

11. __ Asymptotic behavior of a perturbed system, pp. 80-85, International Symposium on Nonlinear Differential Equations and Nonlinear Mechanics, Academic Press, New York, 1963.

12. J. J. Levin, On the global asympotic behavior of nonlinear systems of differential equations, Arch. Rational Mech. Anal. 6 (1960), 65-74.

\section{UNIVERSITY OF WISCONSIN, MADISON, WisCONSIN}

